\title{
Video Technologies: Neologism or Library Trend?
}

Converging factors are shaping a new environment for libraries, and, as a consequence, the present is full of opportunity. Technical and social changes provide libraries with a host of alternatives for service, growth, and innovation.

In this new environment libraries will, undoubtedly, continue to promote the availability of books and other materials, continue to increase their efforts to furnish patrons with information, and continue to broaden the range of activities offered so that patrons can receive personalized service. Patron information seeking and searching methods we have known, however, will give way to new methods based on computers and telecommunications.

A host of new technologies is growing out of the evolutionary pathway marked by telegraph, telephone, radio, and television. Broadband communications (that's the cable that today brings you predominantly entertainment television), satellite, videotex, teletext; videodisc, videotape, large-screen television, and computer displays (some are as large as the side of a building) are available either today or within the next year or two. Each of these technologies is a new medium within its own inherent capabilities and limitations. Each has the promise of providing faster and more cost-efficient information services than some present forms of printed communication. And each requires a different approach and different knowledge for effective and efficient use, and integration into library operations.

In a growing number of locations, cable communications for delivery of library services have already been made available virtually free of charge. Other technologies, such as videotex, will grow significantly. Estimates suggest that in five years more than 8 million American homes will be able to obtain extensive, automated information services from commercial, private, and government sources. Probably a larger number will receive limited information services over the broadcast airwaves via teletext. Dramatic new services will combine television, computer, telephone, satellite, and cable into home entertainment and information centers . . . potential extensions of libraries.

Some sources suggest more than 50 percent of the American Gross National Product results from the collection, processing, and dissemination of information, much of which involves new technologies. Inev- 
itably, this technological trend also will occur in libraries and, in this light, the relatively low level of involvement of computers in providing patron services today is notable. By their natural inertia, individuals and organizations in the library community will be opposed to the acceptance of cable services, videotex, online catalogs, information retrieval, and other video technologies simply because it represents change. But these technologies are technically feasible and are becoming an economic reality. The point of demarcation between computer and library may well become a terminal in a patron's home. Whether or not the service provided is a library's or a commercial competitor's depends to a great extent on how libraries define their role in this environment, and on the degree of library participation in the evolutionary process that's now taking place.

Something besides inertia opposes the acceptance of new technologies, however. To some degree, lack of awareness of technological trends is a factor, but more significant is a lack of clear understanding (both by the proponents of the technology and by librarians) of how new technology can be integrated into the library setting. Understanding the value a technology offers - for increased service or decreased cost, for example - should be paramount, but frequently the technology seems to be offered as an end in itself.

Internal and external factors must be considered to guide the application of technology toward meeting library and patron needs. Financial concerns, social forces, and the consumer/patron appear to be major factors leading libraries toward a future deeply involved in video technologies. Whether the outcome will result from external pressure or internal plan remains to be seen. It's incumbent upon libraries to be informed and active participants in directing their own future in this kind of an environment.

What are the implications of this technical evolution and internal/external factors? One thing is sure: it's a massive industry growing at a very rapid rate, and it is going to grow even faster. Libraries have the opportunity to grow with this trend through application of the technologies to existing technical services, increased availability of patron services, and development of innovative services. If there is a common thread that can identify those libraries which will grow and prosper, that thread is flexibility - the capacity of library management and staff to adapt this library to the new environment, and integrate technology into their library.

Readers and contributors of JOLA are the people that can either have an integral part in defining the future direction of libraries, or passively watch patron needs outstrip services. Library schools and people involved in library-related research must play a key role in assessing the value of video technologies and defining how to integrate them into the business and service of libraries. What is going to preserve and enhance 
the role of libraries in the 1980 s will not only be flexibility but another very critical element - foresight, dedicated to patron needs. Many libraries have met this technological revolution head-on and are intimately involved in testing, developing, and providing innovative library services.

In this and forthcoming issues, we hope to bring a perspective on these changes that is valuable and cogent to the library community. Readers of JOLA and practitioners in all areas of video technology are called upon to describe their efforts and share their results drawn from this rapidly changing field through contributions to this journal.

THOMAS D. HARNISH

\section{EDITOR'S NOTES}

JOLA will continue to be interesting and useful to its readers to the extent that its readers are willing to expend the efforts to also be its writers. The authors in this issue are all as busy as you and I. They have made time in their already full schedules to write down ideas and information they hope will be useful and provocative. They and we of the JOLA staff hope you are pleased with the results.

So what's new by you? How have your patrons reacted to your new online catalog? What do the costs of your acquisitions system look like? How about that idea you had about a new way to do whatever? Do you think the fuss over authority control is worth it? If you have, ideas, perceptions, or stories to tell that you feel are of interest to your fellow readers, please write and let them know. 